\title{
SESGO HACIA EL PRESENTE, FUENTES DE FINANCIAMIENTO Y VARIABLES PRODUCTIVAS: EVIDENCIA DE UN GRUPO DE PRODUCTORES DE LECHE EN HERMOSILLO, SONORA
}

PRESENT BIAS, FINANCIAL SOURCES AND PRODUCTIVE VARIABLES: EVIDENCE OF A GROUP OF MILK PRODUCERS IN HERMOSILLO, SONORA

\author{
Eduardo Leyva León \\ lelehim@gmail.com \\ Carlos Gabriel Borbón Morales \\ cborbon@ciad.mx \\ José Ángel Vera Noriega ${ }^{1}$ \\ avera@ciad.mx \\ Francisco Fernando Durazo Salas ${ }^{2}$ \\ durazof@gmail.com \\ Hermosillo, Sonora México

1 Centro de Investigación en Alimentación y Desarrollo A.C. 
Para citar este artículo:

Leyva-León, Eduardo, Borbón-Morales, Carlos, Durazo-Salas, Francisco. (2019).

Sesgo hacia el presente, fuentes de financiamiento y variables productivas:

evidencia de un grupo de productores de leche en Hermosillo, Sonora. Espacio

I+D, Innovación más Desarrollo. VIII(21), 76-94. doi: http://dx.doi.org/10.31644/

IMASD.21.2019.a05

\section{RESUMEN}

El objetivo de este trabajo es relacionar el sesgo hacia el presente (sHP) con la elección de fuentes de financiamiento y variables productivas de un grupo de productores de leche en Hermosillo, Sonora. Se seleccionó de manera intencional una muestra de 53 productores de leche ( 8 mujeres y 45 hombres) con un rango de edad de 29 a 73 años. A través de un cuestionario socioeconómico, productivo, financiero y una prueba de elección, se encontró que (a) el 51\% de los productores optan por fuentes informales de financiamiento, de los cuales el $72 \%$ presentaron shP; (b) se registró una producción total de leche de 7,128 litros diarios, en donde el 59\% del volumen producido correspondió a productores con SHP; y (c) se obtuvieron diferencias estadísticamente significativas entre el sHP y el tipo de fuente de financiamiento, pero no entre el SHP y la producción. Los resultados obtenidos en la presente investigación no solamente reflejan la alta prevalencia de SHP en los productores, sino que también presentan inconsistencia en sus preferencias, mismas que pueden afectar el desarrollo de la unidad de producción.

\section{Palabras clave}

Sesgo hacia el presente; fuentes de financiamiento; producción de leche: elección intertemporal. 


\section{- Abstract-}

The aim of this work is to relate the present bias ( $\mathrm{PB})$ with financial sources choice and productive variables of a group of milk producers in Hermosillo, Sonora. A sample of 53 milk producers ( 8 women and 45 men) with an age range of 29 to 73 years was intentionally selected. Through a socioeconomic, productive and financial questionnaire and a choice test, it was found that: (a) the $51 \%$ of the producers choose informal sources, of which $72 \%$ presented $\mathrm{PB}$; (b) a total milk production of 7,128 liters per day was registered, where $59 \%$ of the volume produced corresponded to PB producers; and (c) statistically significant differences were found between the Рв and the type of financial source, but not between Рв and production. The results in the present investigation not only reflect the high prevalence of Pв in the producers, but also have inconsistency in their preferences, which may affect the development of the production unit.

\section{Keywords}

Present bias; financial sources; milk production; intertemporal choice. 
$\mathrm{E}$ n México el sector pecuario representa una importante actividad, ya que coadyuva a la producción y oferta de carne y lácteos, estos son considerados como productos básicos y estratégicos para la población (Ley de Desarrollo Rural Sustentable, 2001). Además, tiene un aporte económico significativo al producto interno bruto (PIB) del 3.5\% (INEGI, 2018).

A pesar de la relevancia de esta actividad, existen problemáticas que dificultan el desarrollo de la rama productiva. Dentro de los problemas característicos de los productores agropecuarios a nivel nacional y que es de interés para el presente estudio, está la escasez de recursos económicos. Para hacer frente a esto, los productores buscan fuentes de crédito que les permitan financiar la adquisición de insumos y tener un crecimiento en la producción (Escalante et al., 2013). Por lo tanto, la elección de fuentes de financiamiento se torna importante para los productores, principalmente para los pequeños y medianos.

Sin embargo, en años recientes han proliferado estudios de elección financiera como los de Brown y Previtero (2014); Carvalho et al. (2016); Delaney y Lades (2017); Kuchler y Pagel (2017) y Gill et al. (2018); los cuales han evidenciado preferencias en recibir recompensas inmediatas, por parte de las personas. A esto se le conoce como sesgo hacia el presente (sHP). Este comportamiento se ha manifestado, principalmente, al momento de realizar elecciones financieras tales como el ahorro, inversión y el uso de tarjetas de crédito (endeudamiento).

Por tal motivo, resulta de interés relacionar el shp con las elecciones financieras y las variables de producción y establecer un panorama del comportamiento de los productores. Para comprender el entorno financieroproductivo y la noción del SHP, es necesario iniciar con un recuento de la problemática en el sector pecuario regional y el sustento teórico del SHP, dividido en: (a) contexto productivo del sector pecuario, (b) distribución y elección de financiamiento en el sector agropecuario y (c) la elección intertemporal: una perspectiva desde la economía del comportamiento. Una vez establecido el apartado de desarrollo, se presenta la metodología, el análisis de resultados y las conclusiones.

\section{CONTEXTO PRODUCTIVO DEL SECTOR PECUARIO}

El sector ganadero mexicano ha registrado ciertos cambios, entre estos se encuentra la creciente exportación de carne de cerdo a Japón, caracterizado por procesos agroindustriales altamente tecnificados. Sin embargo, la ganadería bovina se ha mantenido en una dinámica tradicional, vinculada a la exportación de carne a Estados Unidos (Salazar et al., 2011). En cuanto a la participación de los Estados al PIB agropecuario, los que contribuyen con 
mayor proporción son Jalisco (11.3\%), Michoacán (9.4\%), Sinaloa (7.7\%), Veracruz (7.2\%), Chihuahua (6.4\%) y Sonora 5.9\% (SAGARHPA, 2016).

Por otro lado, la producción de leche bovina en México en el 2017 registró volúmenes de 11, 807,556 litros (SIAP, 2018). Esta actividad se desarrolla en condiciones heterogéneas desde las perspectivas económica, técnica, social y ambiental y se constituyen principalmente como empresas familiares (Espinoza et al., 2011).

Mientras que en Sonora la producción de leche se divide en dos formas: con ganado especializado y de doble propósito, en donde el primero produce $32 \%$ y el segundo $67 \%$; y de la producción total de leche, el $58 \%$ se destina a la elaboración de queso y el $42 \%$ restante como leche fresca (Salazar et al., 2011). Esta actividad genera 18 millones de pesos anualmente, beneficiando a más de 33 mil familias en Sonora (sAgARHPA, 2017).

Sin embargo, existen problemáticas que afectan la actividad productiva tales como: apoyos insuficientes y mal distribuidos de las entidades gubernamentales, una falta de regulación de los precios de la leche, el incremento de importación de leche en polvo, así como altos índices de pobreza en zonas rurales (Borbón et al., 2011; Huesca et al., 2011). Por lo tanto, el acceder y elegir una fuente de financiamiento resulta significativo, ya que el crédito es uno de los principales componentes que impulsa el crecimiento de estas organizaciones, puesto que mejora la productividad, la gestión de riesgos y la inclusión productiva (Fletschner y Kenney, 2011; Olluqui y Fernández, 2017).

\section{DISTRIBUCIÓN Y ELECCIÓN DE FINANCIAMIENTO EN EL SECTOR AGROPECUARIO}

La asignación de créditos agropecuarios en México se encuentra relacionada con las entidades que tienen un mayor aporte al PIB agropecuario, y presenta una concentración significativa en Sinaloa, Jalisco y Sonora. En el cuadro 1 se muestra la proporción del financiamiento en estos tres Estados, del periodo 2013 a 2016. En términos generales, el financiamiento se ha caracterizado por una tendencia estancada, ya que ha sido poco el incremento de la proporción del crédito que se ha detectado. Estos Estados se caracterizan principalmente por la producción de hortalizas, maíz, trigo y la actividad ganadera.

\section{Cuadro 1}

Proporción del financiamiento agropecuario por Estados

\begin{tabular}{ccccc}
\hline Estados & $2013(\%)$ & $2014(\%)$ & $2015(\%)$ & $2016(\%)$ \\
Sinaloa & 13.9 & 13.2 & 13 & 13.1 \\
Jalisco & 9.1 & 9.4 & 8.6 & 9.8 \\
Sonora & 7.4 & 6.5 & 6.7 & 6.6 \\
\hline
\end{tabular}

Fuente: Elaboración propia en base a FIRA 2013-2016. 
En el cuadro 2 se presenta la proporción de financiamiento pecuario otorgado por la banca de desarrollo en México, de 2013 a 2016. Se observa que los Fideicomisos Instituidos en Relación con la Agricultura (FIRA) asignan mayor proporción del financiamiento al sector; no obstante, a pesar de que la Financiera Nacional de Desarrollo (FND) se encuentra por debajo de FIRA, su participación en el sector es significativa.

Cuadro 2

Proporción del financiamiento al sector pecuario

\begin{tabular}{ccccc}
\hline Fuente de Financiamiento & $2013(\%)$ & $2014(\%)$ & $2015(\%)$ & $2016(\%)$ \\
FIRA & 20.3 & 19.1 & 21.1 & 22.6 \\
FND & 9.4 & 9.9 & 14.4 & 15.1 \\
\hline
\end{tabular}

Fuente:Elaboración propia en base a FIRA y FND 2013-2016.

Sin embargo, a pesar de la creciente asignación del financiamiento al sector pecuario, el $64 \%$ de los productores recurren a fuentes informales de financiamiento, mientras que el porcentaje restante elige fuentes formales: el $17 \%$ a la banca de desarrollo, 9\% acude a la banca comercial, 9\% a uniones de crédito y el $1 \%$ a Sociedades Financieras de Objeto Limitado (SOFOL) (Escalante et al., 2013). Lo anterior demuestra la existencia creciente de un mercado informal del crédito, así como el financiamiento de proveedores de insumos o servicios (Moreno, 2002).

De acuerdo a Puyana y Romero (2010), este comportamiento de los productores al momento de elegir financiamiento se debe en gran medida, a los costos de transacción por parte de las bancas e instituciones financieras. Dichos costos implican: (a) la disposición de sucursales de financiamiento, (b) los tiempos que el productor emplea para los trámites del crédito, y (c) identificación y disposición de activos (colaterales) (Puyana y Romero, 2007). Con base a lo anterior, un aspecto importante que caracteriza a las fuentes de financiamiento informal es la ausencia de costos de transacción. Esto se ve traducido para el productor en la obtención del financiamiento de manera inmediata, sin embargo las tasas de interés que ofrecen estos son mayores a las del mercado formal (Olivares, 2004).

La elección intertemporal: una perspectiva desde la economía del comportamiento

Este comportamiento de los productores en el contexto financiero, ha sido objeto de estudio de la economía por medio de la elección intertemporal y se han identificado supuestos y modelos a través de los cuales es posible comprender dicho comportamiento. Estas elecciones implican decisiones en 
las cuales la distribución de costos y beneficios se extienden a lo largo del tiempo (Loewenstein y Thaler, 1989). En economía, el modelo utilizado para estudiar las elecciones intertemporales es el de utilidad descontada (UD), el cual tiene sus orígenes en los trabajos de los autores: John Rae, N.W. Senior, William Jevons, Böhm-Bawerk, Irving Fisher y Paul Samuelson (Loewenstein, 1992). Sin embargo, Samuelson (1937) introdujo como tal la UD, cuyo objetivo era modelar las elecciones intertemporales de las personas; y supone que los individuos descuentan eventos futuros a una velocidad constante.

No obstante, en trabajos como el de Strotz (1956) y Phelps y Pollak (1968), se observó que las personas cambian la preferencia de sus elecciones en lapsos de tiempo diferentes. De acuerdo a esto, Strotz (1956) propuso que las preferencias cambian con la distancia temporal, y presentan una inconsistencia. Esto indica que el supuesto de consistencia temporal de la UD no se adecúa a todos los contextos de elección.

A raíz de estos trabajos, surgieron estudios de diseño experimental asociados a la elección intertemporal como los de Thaler $(1981,1988)$ y Loewenstein y Thaler (1989), en donde no solamente se encontró que el comportamiento es guiado por preferencias temporales, sino que también existen ciertas anomalías en las elecciones intertemporales que se contraponen a la UD. Thaler (1988), menciona que una anomalía es un resultado empírico, específicamente una elección efectuada, que es difícil de explicar a través de la racionalidad o que son necesarias suposiciones inverosímiles para su comprensión. Estas anomalías son atribuibles a una limitación en el procesamiento de la información, que las personas cometen al momento de efectuar elecciones. Los esfuerzos realizados por Thaler (1981), así como los de Tversky y Kahneman (1974) y Kahneman y Tversky (1979), dieron origen a la economía del comportamiento, por medio de la evidencia de anomalías en las elecciones de los individuos.

Dentro de las anomalías que corresponden a las elecciones intertemporales, el sesgo hacia el presente (sHp) es la que concierne a este estudio, ya que en los financiamientos se establecen plazos (presente vs. futuro), y el shp es una tendencia por parte de los individuos a dar un mayor peso a las recompensas que se encuentran más cercanas al tiempo actual, al considerar intercambios en dos momentos en el tiempo (presente y futuro) (O’Donoghue y Rabin, 1999). Además, el shp es relevante debido a que actúa como indicador de las elecciones intertemporales (Meier y Sprenger, 2010).

El shp, también llamado descuento-cuasi hiperbólico, es representado por un modelo en donde se observa que los individuos presentan un sesgo por la inmediatez. Éste se ha basado en los aportes realizados por Strotz (1956); Phelps y Pollak (1968) y Laibson (1997), en donde se introdujo un factor adicional a la función que representa al shp (Patiño y Gómez, 2015), y se encuentra dado por: 


$$
U^{t}\left(u_{t}, u_{t+1}, \ldots, u_{T}\right)=\delta^{t} u_{t}+\beta \sum_{T=t+1}^{T} \delta^{T} u_{T}
$$

Donde $0<\beta, \delta \leq 1$. En este modelo $U$ representa las utilidades, $\delta$ el descuento consistente en el largo plazo. Mientras que $\beta$ representa el shp, si se encuentra cercano a uno significa que el sesgo es mínimo, por otro lado, si $\beta<1$ existe un peso mayor por recibir gratificaciones inmediatas por parte de los individuos; por lo tanto, están más sesgados hacia el presente ( $\mathrm{O}^{\prime}$ Donoghue y Rabin, 1999). Pero si $\beta>1$ se atribuye un peso menor a las recompensas inmediatas, por lo tanto las personas están sesgadas hacia el futuro. Sin embargo, si $\beta=1$ entonces el modelo se regresaría al descuento exponencial del modelo de uD, esto quiere decir que los individuos son consistentes en sus elecciones.

En estudios como los de Laibson (1997); Fehr (2002); Heidhues y Koszegi (2010) y Meier y Sprenger (2010); se observa una relación del shP con un alto endeudamiento financiero. En Can y Erdem (2013) y Carvalho et al. (2016), relacionan el SHP con recursos financieros, argumentando que los individuos se comportan con shp cuando sus recursos financieros son limitados. Mientras que en Mani et al. (2013), quienes llevaron a cabo un estudio con productores de caña, encontraron que los participantes enfocan más su atención en situaciones inmediatas, esto propicia que adquieran financiamientos con mayor frecuencia. A pesar de la existencia de diversos estudios que vinculan el shp con el entorno financiero, no se ha encontrado evidencia empírica que relacione el sHp con las fuentes de financiamiento.

Por otro lado, en relación al shp y la producción, en el estudio de Liebenehm y Waibel (2014), realizado con productores pecuarios en África Occidental, encontraron que los participantes presentaron niveles altos de paciencia (bajo SHP); además, identificaron que factores como el tamaño del hato (número de cabezas de ganado) y los ingresos por ventas de productos derivados de la ganadería, influyen en las preferencias temporales. Mientras que Pushkarskaya y Marshall (2009), llevaron a cabo un estudio con productores de tabaco en Kentucky, y encontraron relación entre las opciones de elección y la decisión de salir del mercado de tabaco.

Con base a lo establecido anteriormente, el objetivo de este estudio es relacionar el sHP con la elección de fuentes de financiamiento y las variables productivas de un grupo de pequeños y medianos productores de leche en Hermosillo, Sonora, México. 


\section{METODOLOGÍA}

\section{Diseño y muestra}

Se utilizó un diseño cuasi-experimental, debido a que se trabajó con un grupo de productores intacto, lo que implica una carencia en la aleatorización de los participantes y un control parcial de variables externas (Campbell y Stanley, 1995); además se modifica la variable independiente (Recompensas monetarias y el tiempo en recibirlas), para conocer su efecto en las elecciones de los participantes. Se seleccionó de manera intencional una muestra de 53 productores de leche de Hermosillo, Sonora, México ( 8 mujeres y 45 hombres) en un rango de edad de entre 29 y 73 años. El nivel de estudio que predomina en los participantes es primaria y secundaria, ambos con el $26 \%$.

\section{Instrumento y medidas}

El instrumento se divide en cuatro secciones: (a) variables sociodemográficas: Edad, Sexo y Nivel de Estudios; (b) variables productivas: Hectáreas, Cabezas de ganado en producción, Producción de leche e Ingresos por venta de leche; (c) variables de elección de fuentes de financiamiento: tipo de fuente de financiamiento y último monto financiado que se otorgó; y (d) el tratamiento experimental: una prueba de elección intertemporal. La prueba de elección (choice task) fue seleccionada como instrumento ya que proporciona los tipos de sesgo que se pueden presentar al momento de elegir. Además esta prueba es la más utilizada por los autores que trabajan en este tema (Can y Erdem, 2013; Carvalho et al., 2016; Meier y Sprenger, 2010; Nguyen, 2016).

Una vez que los participantes respondieron a los apartados socioeconómico, productivo y de financiamiento, realizaron la prueba de elección utilizando un incentivo monetario hipotético. La prueba consta de 19 elecciones divididas en tres bloques: $(\mathrm{a})$ to $=$ presente vs $\mathrm{t} 1=1 \mathrm{mes},(\mathrm{b})$ to $=$ presente vs $\mathrm{t} 6=6$ meses, $\mathrm{y}$ (c) $\mathrm{t} 6=6$ meses vs $\mathrm{t} 7=7$ meses; en cada una se presenta una recompensa menor en los tiempos más cercanos al presente, que van de MXN\$1,000 a MXN\$1,550. Mientras que en los periodos más alejados al presente se establece una mayor recompensa fija con valor de MXN\$1,600. Utilizando la información producida por los diferentes puntos en el tiempo (presente y futuro), se obtiene una medida de Factor de Descuento Individual (IDF), la cual no solo permite la identificación del tipo de descuento (exponencial o cuasi-hiperbólico), sino también la identificación de la existencia de shp, sesgo hacia el futuro o consistencia (Meier \& Sprenger, 2010).

El IDF se obtiene cuando en la prueba se observa un punto de cambio (switching point) en la elección; esto es, cambiar de elegir la recompensa 
pequeña a elegir la recompensa grande. Por ejemplo, si un individuo prefiere MXN\$1,550 hoy sobre MXN\$1,600 en un mes, pero prefiere MXN\$1,600 en un mes sobre MXN\$1,400 hoy, entonces se toman los MXN\$1,550 como punto de cambio y se calcula el factor de descuento $(1,550 / 1,600)=0.968$.

Con base al IDF se calcula el SHP; es decir, si una persona es más paciente (tiene un bajo IDF) cuando elige una recompensa pequeña y cercana en el tiempo $(t=0)$; entonces se considera que el individuo es sesgado hacia el presente $\operatorname{si} I D F_{0,1}<I D F_{6,7}$, y es sesgado hacia el futuro si $I D F_{0,1}>I D F_{6,7^{\circ}}$ Cuando una persona se encuentra sesgada hacia el presente es considerada como dinámicamente inconsistente (inconsistente en sus elecciones). Mientras que para obtener los parámetros $\beta$ y $\delta$, se utilizaron las siguientes medidas de IDF de acuerdo a Meier y Sprenger (2010): $\delta=I D F_{6,7} ; \beta=I D F_{0,1} / I D F_{6,7}$. Para realizar la regresión no lineal se utilizó el modelo de descuento cuasi-hiperbólico $\left\{1, \beta \delta, \beta \delta^{2}, \beta \delta^{3}, \ldots\right\}$ (Laibson, 1997; O`Donoghue y Rabin, 1999).

Un resultado importante relacionado con el sesgo es el radio. El cual indica la intensidad del sesgo: en el caso de shP el radio será mayor que 1 (ej. 1.01, 1.06, 1.10), entre más alejado de 1 sea el radio la intensidad del sHP es mayor. Por otro lado, cuando hay sesgo hacia el futuro será menor que 1 (ej. 0.986, 0.902, 0.877), entre más alejado de 1 mayor será el sesgo hacia el futuro. Mientras que, cuando el radio sea igual a 1 habrá consistencia, esto indica que la persona se comporta como lo menciona el descuento exponencial de la utilidad descontada (Meier y Sprenger, 2010).

\section{Análisis de datos}

En este estudio se realizó un análisis descriptivo básico de las variables, para posteriormente realizar pruebas de relación. Se utilizó la prueba estadística t de Student para establecer la existencia de diferencias estadísticas entre el tipo de fuente de financiamiento y el SHP. Se empleó la prueba Ji Cuadrada para establecer la relación entre el tipo de sesgo y la fuente de financiamiento. Además se aplicó la prueba ANDEva de un factor para las variables de tipo de sesgo, número de cabezas de ganado y la producción de leche. Las pruebas se realizaron en el software spss v2o. Asimismo, se ajustó la curva cuasi-hiperbólica para los productores sesgados hacia el presente, en relación al tipo de fuente de financiamiento que eligieron. Para graficar la curva se utilizó el software GraphPad Prism 6. 


\section{RESULTADOS}

\section{Estadística descriptiva}

El apartado de análisis de resultados se encuentra dividido en cinco secciones: (a) frecuencias obtenidas de las variables socioeconómicas, (b) frecuencias obtenidas de las variables productivas, (c) frecuencias y porcentajes de las variables de financiamiento, (d) frecuencias y porcentajes de la elección intertemporal, y (e) análisis comparativo de las variables.

El promedio de edad de los productores es de $57 \pm 11.8$, en donde predomina con un $85 \%$ (45) el sexo masculino y $15 \%$ el femenino (8). En cuanto al nivel de estudios, el $58 \%$ de los productores cuenta con educación básica solamente, $27 \%$ tienen preparatoria y el 15\% estudios superiores. El $46 \%$ tiene un dependiente solamente.

En cuanto a la sección relacionada con la producción, el promedio de hectáreas con las que cuentan las unidades de producción de los participantes fue de $213 \pm 172$. El número total de cabezas de ganado registrado fue de 2,407 , con un promedio por productor de $45 \pm 25$. Mientras que en la producción de leche se observó que en promedio un productor obtiene $134.5 \pm 79.5$ litros al día. En cuanto al precio de venta por litro de leche, el promedio es de MXN\$6.30, lo cual implica que los productores reciban en promedio MXN\$852 \pm 526 al día.

En el apartado financiero, en cuanto al tipo de intermediario que eligieron los productores, se observó ligeramente una mayor preferencia por las fuentes informales de financiamiento, con un $51 \%$, mientras que el $49 \%$ restante optaron por fuentes formales. Con respecto a este resultado, del porcentaje de productores que eligió fuentes formales el 30\% correspondió a la Banca Privada y el 19\% a la Banca de Desarrollo; por el lado de los productores que prefirieron fuentes informales, el $43 \%$ eligió a prestamistas (particulares) y el $8 \%$ restante se encasilló en otro, englobando a proveedores de insumos.

Otro dato importante es el último monto que se otorgó a los productores, en donde el promedio de financiamiento fue de MXN\$68,396. Esta cifra es elevada debido a que los financiamientos por parte de las fuentes formales (Banco privado y Financiera Nacional de Desarrollo) resultaron montos altos, tal y como se observa en el cuadro 3.

\section{Cuadro 3}

Montos financiados en relación al tipo de intermediario financiero

\begin{tabular}{cccc}
\hline $\begin{array}{c}\text { Último monto Financiado } \\
(\text { MXN) }\end{array}$ & Banca Privada & $\begin{array}{c}\text { Financiera Nacional } \\
\text { de Desarrollo }\end{array}$ & Fuente Informal \\
3,000 a 16,900 & $13 \%(7)$ & - & $51 \%(27)$
\end{tabular}




\begin{tabular}{cccc}
17,000 a 50,000 & $17 \%(9)$ & - & - \\
51,000 a 100,000 & - & $6 \%(3)$ & - \\
101,000 a $1,000,000$ & - & $13 \%(7)$ & - \\
\hline
\end{tabular}

Fuente: Elaboración propia con datos de campo. Nota: El número de observaciones se encuentra en paréntesis.

Como se muestra en el cuadro 3, el 16\% de los productores que eligieron a la Financiera Nacional de Desarrollo, se les otorgó un financiamiento que asciende los MXN\$51,000 y MXN\$100,000 principalmente. Esto conlleva a que la media del monto financiado sea elevada. Sin embargo, al aplicar la mediana a esta variable, se obtuvo un monto de MXN\$12,000.

Por otro lado, en relación a las variables obtenidas en la prueba de elección, es importante empezar con la identificación del IDF, en el cual se obtuvo una media de $.865 \pm .09$. A partir de este valor, es posible realizar una clasificación de las preferencias temporales de los productores, donde se presenta la proporción en porcentaje de la preferencia en el tiempo de los productores.

El 60\% de los productores presentó una preferencia temporal con SHP, esto significa que muestra avidez por recibir recompensas económicas lo más cercanas al tiempo presente, sin importar que en el futuro exista una recompensa mayor. El 15\% se encontró sesgado hacia el futuro, lo que nos indica que estos productores se encuentran más preocupados por recibir recompensas económicas en un futuro. Ambos tipos de sesgo son considerados como dinámicamente inconsistentes, ya que no mantienen una consistencia entre sus preferencias presentes con las futuras. Por otro lado, el 25\% fueron dinámicamente consistentes, lo cual significa que sus preferencias son consistentes entre dos puntos en el tiempo.

En cuanto al radio, la media fue de $1.07 \pm .17$, esto significa que la intensidad del sesgo está ligeramente inclinada hacia el sesgo hacia el presente. Si el Radio > 1 la intensidad está inclinada hacia el sesgo hacia el presente. Por otro lado, si el Radio < 1 la intensidad estará proclive al sesgo hacia el futuro. Entre más alejado del 1 se encuentre el radio, ya sea a favor del sesgo hacia el presente o futuro, mayor intensidad existirá.

\section{Comparación de las variables}

Una vez presentados los resultados descriptivos de las variables, se muestra el cuadro 4. En ella se establece la relación de los productores que se encuentran sesgados hacia el presente, sesgados hacia el futuro y consistentes; en relación con las variables productivas, de financiamiento y elección intertemporal. 


\section{Cuadro 4}

Medidas descriptivas para las variables productivas, de financiamiento y elección intertemporal en relación a los grupos de productores (sesgados hacia el presente, sesgados hacia el futuro y consistentes)

\begin{tabular}{|c|c|c|c|}
\hline & & & \\
\hline & $\begin{array}{l}\text { Productores Sesgados } \\
\text { hacia el presente }\end{array}$ & $\begin{array}{l}\text { Productores Sesgados } \\
\text { hacia el futuro }\end{array}$ & $\begin{array}{l}\text { Productores } \\
\text { Consistentes }\end{array}$ \\
\hline \multicolumn{4}{|l|}{$\begin{array}{c}\text { A. Variables } \\
\text { Sociodemográficas }\end{array}$} \\
\hline Edad & $\begin{array}{c}56.3(11.7) \\
{[32]}\end{array}$ & $\begin{array}{c}57.4(15.1) \\
{[8]}\end{array}$ & $\begin{array}{l}58.9(10.9) \\
{[13]}\end{array}$ \\
\hline Sexo (1=Masc.) & $0.78(0.42)$ & 1 & $0.92(0.27)$ \\
\hline $\begin{array}{l}\text { Educación (Básica }=0 \\
\text { Media y Sup. }=1 \text { ) }\end{array}$ & $0.40(0.49)$ & $0.5(0.53)$ & $0.38(0.50)$ \\
\hline \multicolumn{4}{|l|}{ B. Variables Productivas } \\
\hline Hectáreas & $228(203)$ & 152 (139) & $215(90)$ \\
\hline $\begin{array}{c}\text { Cabezas de Ganado en } \\
\text { Producción }\end{array}$ & $47(28)$ & $49(27)$ & $38(17)$ \\
\hline $\begin{array}{l}\text { Producción de Leche } \\
\text { Diaria (Its.) }\end{array}$ & $130(79)$ & $170(87)$ & $122.5(76)$ \\
\hline $\begin{array}{l}\text { Ingresos por Venta de } \\
\text { Leche (MXN) }\end{array}$ & $854(528)$ & $1,231(764)$ & $837(612)$ \\
\hline \multicolumn{4}{|l|}{$\begin{array}{l}\text { B. Variables de } \\
\text { Financiamiento }\end{array}$} \\
\hline $\begin{array}{l}\text { Fuente de Financiamiento } \\
\text { (Informal }=0 \\
\text { Formal }=1)\end{array}$ & $0.28(0.45)$ & $0.87(0.35)$ & $0.77(0.43)$ \\
\hline $\begin{array}{l}\text { Último Monto Financiado } \\
(M X N)\end{array}$ & $\begin{array}{c}77,328 \\
(243,256)\end{array}$ & $\begin{array}{c}51,375 \\
(43,506)\end{array}$ & $\begin{array}{c}56,884 \\
(79,563)\end{array}$ \\
\hline \multicolumn{4}{|l|}{$\begin{array}{l}\text { C. Variables de Elección } \\
\text { Intertemporal }\end{array}$} \\
\hline IDF & $0.875(0.07)$ & $0.850(0.14)$ & $0.849(0.10)$ \\
\hline Radio & $1.15(0.13)$ & $0.85(0.13)$ & 1 \\
\hline
\end{tabular}

Fuente: Elaboración propia con base a los datos recabados en la investigación.

Nota: La tabla muestra medias y frecuencias, desviación estándar en paréntesis y el número de observaciones en corchetes.

Con respecto a los productores con SHP, el 60\% se ubicó en este grupo, el cual se caracterizó por tener una edad de 56 años, predominó el sexo masculino y el nivel de estudios fue de educación básica. En el apartado productivo, en promedio cuentan con 228 hectáreas, poseen 47 cabezas de ganado en producción, producen 130 litros de leche al día y reciben en promedio MXN\$854 por la venta de la leche. En cuanto a las variables de financiamiento de los productores con SHP, el promedio eligió fuentes informales y el último monto financiado fue de MXN\$77,328. Es importante mencionar que la cifra del monto financiado no es exclusiva para las fuentes informales, más bien es para los productores que se encuentran sesgados 
hacia el presente. Ya que existen productores sesgados hacia el presente que eligieron fuentes formales de financiamiento y por lo tanto el monto financiado asciende a mayores cifras. Por otro lado, en las variables de elección intertemporal, la media del IDF del grupo con shP fue de $0.876 \pm 0.07 \mathrm{y}$ el radio de $1.15 \pm 0.13$, ambos superiores al promedio total de productores. En cuanto al promedio del factor de descuento de los productores con SHP, el parámetro fue $\delta=0.876$ y $\beta=0.870$.

En los productores sesgados hacia el futuro, el promedio de edad fue de 57 años, prevaleció el sexo masculino, el nivel de estudios fue de $50 \%$ educación básica y el 50\% restante educación media y superior. En promedio cuentan con 152 hectáreas, muy por debajo de la media total; poseen 49 cabezas de ganado, producen 170 litros de leche diario y reciben MXN\$1,231 en promedio. En cuanto a su condición de financiamiento, éstos eligieron en promedio fuentes formales y el último monto financiado fue de MXN\$51,375. Referente a la elección intertemporal, los productores sesgados hacia el futuro presentaron un IDF medio de $0.850 \pm 0.14 \mathrm{y}$ un radio de $0.850 \pm 0.13$.

Mientras que los productores consistentes, la edad promedio fue de 59 años, el sexo masculino siguió prevaleciendo, el nivel de estudios fue de educación básica. En el ámbito productivo tienen en promedio 215 hectáreas, 38 cabezas de ganado, producen 122.5 litros de leche diario y reciben MXN\$837 al día. En su estado financiero predominaron las fuentes formales de financiamiento y el último monto financiado fue de $\mathrm{MXN \$ 56,884}$. Respecto a la elección intertemporal la media del IDF fue de $0.849 \pm 0.10$ y el radio de 1 .

Un resultado importante que se observa en el cuadro 4, es en relación a los grupos de productores sesgados hacia el presente, futuro y consistente, con la producción de leche. Ya que los sesgados hacia el futuro producen mayor cantidad de leche (170lts.), después se encuentran los shP (13olts.) y los consistentes (122.5lts.). De igual forma, los productores sesgados hacia el futuro recibieron mayores ingresos por la venta de su producción (MXN\$1,231). Sin embargo, no se encontraron diferencias significativas entre el tipo de sesgo y el último monto financiado $\mathrm{F}(2)=0.985, \mathrm{p}=0.38$; ni entre el tipo de sesgo e ingresos por venta de leche $F(2)=0.911, p=0.40$

En cuanto a la relación entre las variables tipo de sesgo (presente, futuro y consistente) y fuente de financiamiento, que se observa en el cuadro 4 , los productores con SHP mostraron una mayor inclinación hacia la elección de fuentes informales de financiamiento. Por otro lado, los que se encuentran sesgados hacia el futuro y los consistentes prefirieron fuentes formales. Se constató que hay diferencias estadísticamente significativas entre el tipo de sesgo y fuente de financiamiento $\chi^{\wedge} 2(2, N=53)=14.3, p=0.001$.

Mientras que en la figura 1 se presentan las medias de los puntos de cambio del factor de descuento de los productores con sHP, relacionado con 
el tipo de fuente de financiamiento. En donde $\beta=0.82$ y $\delta=0.80$ para los que eligieron fuentes informales, mientras que para los que eligieron fuentes formales fue de $\beta=0.88$ y $\delta=0.90$.

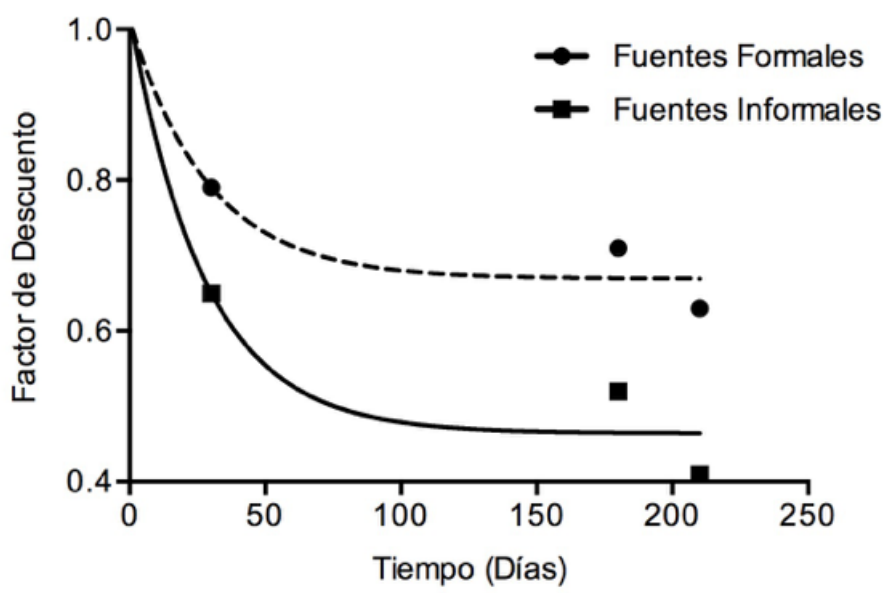

Figura 1. Comparación del tipo de fuente de financiamiento en relación al SHP. Fuente: Elaboración propia.

En la figura 1 se muestra que los productores descontaron el valor de la recompensa conforme aumentó la demora de entrega en la prueba de elección. Sin embargo, en el caso de los productores que eligieron fuentes informales (cuadros), descontaron el valor de manera más rápida, de 1 a 0.65 en un mes; mientras que los productores que eligieron fuentes formales de financiamiento (círculos), fue de 1 a 0.80 en el mismo mes. Por otro lado, la tasa de descuento para los productores que eligieron fuentes informales fue similar $\left(\mathrm{k}=0.036, \mathrm{R}^{\wedge} 2=0.96\right)$ a la tasa de descuento de los que eligieron fuentes formales $\left(\mathrm{k}=0.034, \mathrm{R}^{\wedge} 2=0.95\right)$. Se encontraron diferencias estadísticamente significativas entre el SHP y los productores que eligieron fuentes informales de financiamiento $\mathrm{t}(51)=-1.99, \mathrm{p}=0.05$.

\section{DISCUSIÓN Y CONCLUSIONES}

Los resultados en esta investigación, aportan evidencia al campo de la economía del comportamiento; ya que existen diversos estudios que tratan el sHP o las inconsistencias en la elección intertemporal y el endeudamiento de tarjetas de crédito; sin embargo, no se han encontrado estudios que aborden el shP con respecto a los tipos de fuentes de financiamiento. Por otro lado, en relación al contexto agropecuario, existe escasa evidencia empírica que relacione elementos de preferencias temporales, como el sHP y variables productivas.

De acuerdo a las elecciones intertemporales, los productores exhibieron un mayor shp (60\%), en relación al sesgo hacia el futuro y consistencia. 
Estos resultados se adecúan con los estudios de Kahneman y Frederick (2001); Tanaka et al. (2010); Can y Erdem (2013) y Carvalho et al. (2016), en donde los participantes presentaron preferencias intuitivas y sHP al momento de realizar sus elecciones. Además, de los participantes con sHP, el $72 \%$ eligió fuentes informales de financiamiento sobre las fuentes formales. Sin embargo, no existe un estudio similar que pueda coadyuvar al sustento de lo obtenido, no obstante, en el trabajo de Meier y Sprenger (2010), se encontró que los individuos con un comportamiento SHP tienen probabilidades significativamente altas de endeudarse con créditos financieros. Mientras que Mani et al. (2013) observaron que los productores de caña en la India, se enfocaban en situaciones financieras inmediatas, sobre todo cuando los recursos económicos eran escasos. Si bien es cierto, estos estudios no son similares a la presente investigación, permiten establecer un sustento empírico importante para fundamentar los resultados obtenidos.

En cuanto al IDF, encontramos que el promedio del factor de descuento fue de 0.86 para el total de los productores. Este resultado se adecúa con los obtenidos por Meier y Sprenger (2010), en donde se observó un IDF de o.83. El factor de descuento en nuestro estudio es bajo, sin embargo, es ligeramente mayor al de los autores citados. Mientras que el radio promedio encontrado fue de 1.07, este resultado es inferior en comparación con Meier y Sprenger (2010) (1.26), lo cual indica que la intensidad del shp en los productores es mínima en comparación con el estudio de estos autores. En cuanto a la función cuasi-hiperbólica, se descontó con mayor rapidez en el tiempo con relación a la función exponencial. Esto indica que hay un mayor nivel de impaciencia en los productores que eligieron fuentes informales de financiamiento $(\beta=0.82)$, por recibir recompensas inmediatas, que los que eligieron fuentes formales $(\beta=0.88)$. Los resultados obtenidos concuerdan con los establecidos en Laibson (1997); Berns, Laibson y Loewenstein (2007); Can y Erdem (2013) y Vanderveldt, Oliveira y Green (2016); sin embargo, en estos estudios los valores de $\beta$ son ligeramente más bajos que los presentados en este trabajo, salvo en Can y Erdem (2013), ellos registraron un promedio de $\beta=.955$. Mientras que Liebenehm y Waibel (2014) presentaron valores más bajos $(\beta=.788)$ en productores con SHP.

En cuanto a los resultados de tipo de sesgo y producción, estos coinciden con lo establecido con Pushkarskaya y Marshall (2009) y Liebenehm y Waibel (2014), ya que ellos encontraron un comportamiento shP en productores agropecuarios. Sin embargo, ellos identificaron que los sujetos que contaban con gran número de cabezas de ganado y mayores ingresos, resultaron con mayor SHP. Mientras que en nuestro estudio, los productores que registraron mayor número de cabezas de ganado e ingresos fueron los sesgados hacia el futuro. Esto permite entender la existencia consistente de comportamientos sesgados. 
Los resultados expuestos en este trabajo permiten a las instituciones financieras, privadas o de gobierno, conocer el comportamiento de los agentes frente a elecciones económicas y la influencia que dichas elecciones tienen sobre la producción. En el caso particular de los pequeños y medianos productores lecheros, donde el financiamiento es un factor esencial para su actividad productiva, el elegir de forma adecuada un crédito es vital para el mantenimiento de la cadena productiva.

Dentro del estudio existen ciertas limitantes metodológicas, mismas que se establecen a continuación: (a) debido al tamaño de la muestra en el presente estudio, solamente se presenta la relación entre el shp, los productores que eligen fuentes informales de financiamiento y la producción de leche, por lo tanto, no se permite realizar una inferencia causal del sesgo sobre las fuentes y la producción. (b) Se establece un diseño cuasi-experimental debido a que el estudio involucra una prueba de elección y se realiza en un entorno de campo. Esto quiere decir que no se efectúa en un laboratorio en donde se tienen en control todas las variables. Sin embargo, a pesar de la limitante de no contar con un control total de variables, este tipo de experimentos de campo ayudan a explicar el comportamiento real de las personas al encontrarse en su entorno natural (Cárdenas, 2004). (c) En la prueba de elección del presente estudio se utilizaron recompensas monetarias hipotéticas. Si bien es cierto, la limitante de no utilizar recompensas reales puede afectar la elección de los participantes. Sin embargo, Locey et al. (2011) en su estudio mencionan que se pueden obtener los mismos resultados tanto para las recompensas reales como para las hipotéticas, por lo tanto los experimentos con recompensas hipotéticas se pueden aplicar al comportamiento de la vida cotidiana. 


\section{REFERENCIAS}

Berns, G., Laibson, D. y Loewenstein, G. (2007). Intertemporal choice-toward an integrative framework. Trends in Cognitive Sciences, 11(11), 482-488. doi: https://doi.org/10.1016/j.tics.2007.08.011

Borbón, C., Salido, P., López, M., Romo, E., Bañuelos, N., Romero, D. y Wong, P. (2011). Problemática relevante en el medio rural del estado de Sonora. En C. Borbón (Ed.), Diagnóstico del sector agropecuario y pesquero del Estado de Sonora (pp. 97-168). México: CIAD, SAGARHPA y SAGARPA.

Brown, J. y Previtero, A. (2014). Procastination, Present-Biased Preferences, and Financial Behaviors. En The 16th Annual Joint Meeting of the Retirement Research Consortium. Washington, DC.

Campbell, D. y Stanley, J. (1995). Diseños experimentales y cuasiexperimentales en la investigación social. Buenos Aires: Amorrortu.

Can, B. y Erdem, O. (2013). Income groups and long term investment. Economics Bulletin, 33(4), 3014-3022.

Cárdenas, C. (2004). Bringing the lab to the field: More than changing subjects. Artefactual Field Experiments 00024, The Field Experiments Website.

Carvalho, L., Meier, S. y Wang, S. (2016). Poverty amd economic decisionmaking: Evidence from changes in financial resources at payday. American Economic Review, 106(2), 260-284. doi: https://doi.org/10.1257/aer.20140481

Delaney, L. y Leades, L. (2017). Present bias and everyday self-control failures: A day reconstruction study. Behavioral Decision Making, 30(5), 11571167. doi: https://doi.org/10.1002/bdm.2031

Escalante, R., Catalán, H. y Basurto, S. (2013). Determinantes del crédito en el sector agropecuario mexicano: un análisis mediante un modelo Probit. Cuadernos de Desarrollo Rural, 10(71), 101-124.

Espinosa, V., Jiménez, R., Gil, G., Alonso, A., Brunett, L. y García, L. (17 de diciembre de 2011). Lechería Familiar. La jornada del campo, 51. Recuperado de: http://www.jornada.com.mx/2011/12/17/cam-lecheria.html

Fehr, E. (2002). The economics of impatience. Nature, 415, 269-72. doi: https://doi.org/10.1038/415269a

FIRA (2013-2016). Informe de Actividades 2013 a 2016. México: FIRA.

FND (2013-2016). Informe de Autoevaluación de Gestión de Financiera Nacional de Desarrollo Agropecuario, Rural, Forestal y Pesquero 2013 a 2016. México: FIND.

Fletschner, D. y Kenney, L. (2011). Rural women's access to financial services: Credit, savings and insurance. ESA Working Paper (11-07).

Gill, A., Hett, F. y Tischer, J. (Julio, 2018). Measuring time inconsistency using financial transaction data. SAFE Policy Latter, 71. Recuperado de: https:// safe-frankfurt.de/fileadmin/user_upload/SAFE_Policy_Letter_71.pdf 
Heidhues, P. y Koszegi, B. (2010). Exploiting naivete about self-control in the credit market. American Economic Review, 100(5), 2279-2303. doi: https://doi.org/10.1257/aer.100.5.2279

Huesca, L., Borbón, C. y Robles, A. (2011). La población afectada por la problemática relevante en el sector rural sonorense. En C. Borbón (Ed.), Diagnóstico del sector agropecuario y pesquero del Estado de Sonora (pp. 169-268). México: CiAD, SAGARHPA Y SAGARPA.

INEGI (2018). PIB y Cuentas Nacionales. Recuperado de: http://www.inegi. org.mx/est/contenidos/proyectos/cn/

Kahneman, D. y Frederick, S. (2001). Representativeness Revisited: Attribute Substitution in Intuitive Judgement. En T. Gilovich, D. Griffin \& D. Kahneman (Eds.), Heuristics and Biases: The Psychology of Intuitive Judgment (pp. 49-81). Cambridge: Cambridge University Press.

Kahneman, D. y Tversky, A. (1979). Prospect Theory: an analysis of decision under risk. Econometrica, 42(2), 263-291. doi: https://doi. org/10.2307/1914185

Kuchler, T. y Pagel, M. (2017). Sticking to your Plan: The Role of Present Bias for Credit Card Paydown. NBER Working Paper, 24881. Cambridge: The National Bureau of Economic Research.

Laibson, D. (1997). Golden eggs and hyperbolic discounting. The Quarterly Journal of Economics, 112(2): 443-477. doi: https:/doi. org/10.1162/o03355397555253

Ley de Desarrollo Rural Sustentable (2001). Capítulo XVII De la Seguridad y Soberanía Alimentaria, Artículo 179. Recuperado de: https://www.juridicas.unam.mx/legislacion/ordenamiento/ ley-de-desarrollo-rural-sustentable\#340366.

Liebenehm, S. y Waibel, H. (2014). Simultaneous estimation of risk and time preferences among small-scale cattle farmers in West Africa. American Journal of Agricultural Economics, 96(5), 1420-1438. doi: https://doi.org/10.1093/ajae/aau056

Locey, M., Jones, B. y Rachlin, H. (2011). Real and hypothetical rewards. Judgement and Decision Making, 6(6), 552-564.

Loewenstein, G. (1992). The fall and rise of psychological explanations in the economics of intertemporal choice. En G. Loewenstein y J. Elster (Eds.), Choice over Time (pp. 3-34). New York: Russell Sage Foundation.

Loewenstein, G. y Thaler, R. (1989). Anomalies: Intertemporal choice. The Journal of Economic Perspectives, 3(4), 181-193. doi: https://doi. org/10.1257/jep.3.4.181

Mani, A., Mullainathan, S., Shafir, E. y Zhao, J. (2013). Proverty impides cognitive function. Science, 341(6149), 976-980. doi: https://doi. org/10.1126/science.1238041 
Meier, S. y Sprenger, C. (2010). Present-Biased Preferences and Credit Card Borrowing. American Economic Journal: Applied Economics, 2(1), 193210. doi: https://doi.org/10.1257/app.2.1.193

Moreno, J. (2002). Las finanzas en las empresa: información, análisis, recursos, planeación y reestructuración de las entidades. México: Compañía Editorial Continental S.A.

Nguyen, Q. (2016). Linking loss aversion and present bias with overspending behavior of tourist: Insights from a lab in the field eperiment. Tourism Management, 53, 152-159. doi: https://doi.org/10.1016/j. tourman.2015.09.019

O’Donoghue, T. y Rabin, M. (1999). Doing It Now or Later. The American Economic Review, 89(1), 103-124. doi: https://doi.org/10.1257/ aer.89.1.103

Olivares, J. (2004). El financiamiento informal en el sector agrícola. Visión General, 3(2), 37-45.

Olluqui, F. y Fernández, M. (2017). Financiamiento del sector agroalimentario y desarrollo rural. Banco Interamericano de Desarrollo. Recuperado de: https:// publications.iadb.org/bitstream/handle/11319/8252/Financiamiento-delsector-agroalimentario-y-el-desarrollo-rural.pdf

Patiño, D. y Gómez, F. (2015). ¿Explican las preferencias cuasi-hiperbólicas la procastinación académica? Una evaluación empírica. Woeking Papers - Serie EC (03). doi: http://dx.medra.org/10.12842/w PASEC-2015-03

Phelps, E. y Pollak, R. (1968). On second-best national saving and gameequilibrium growth. The Review of Economic Studies, 35(2), 185-199. doi: https://doi.org/10.2307/2296547

Pushkarskaya, H. y Marshall, M. (2009). Lump sum versus annuity: Choices of Kentucky farmers during the Tobacco Buyout Program. Journal of Agricultural and Applied Economics 41(3), 613-624. doi: https://doi. org/10.1017/S1074070800003102

Puyana, A. y Romero, J. (2007). La revaluación cambiaria y el retroceso del sector agropecuario mexicano. ¿Una historia de medio siglo? En M. Mántey y N. Levy (Coords.), Políticas Macroeconómicas para Países en Desarrollo (pp. 139-178). México: UnAM.

Puyana, A. y Romero, J. (2010). Estancamiento del sector agropecuario mexicano. Textual - análisis del medio rural latinoamericano, (53), 29-62.

SAgarhPa. (2016). Producto Interno Bruto por Actividades. Recuperado de: http://oiapes.sagarhpa.sonora.gob.mx/notas/econo/pib-2016.pdf

SAGARHPA. (2017). Información del Sector Agropecuario, Pesquero y Acuícola del Estado de Sonora. Recuperado de: http://oiapes.sagarhpa.sonora.gob. $\mathrm{mx} / \mathrm{edo1}$.pdf

Salazar, V., Moreno, M. y Arvizu, M. (2011). El sector rural del estado de Sonora: recursos naturales, demografía y estructuras agropecuaria y 
pesquera. En C. Borbón (Ed.), Diagnóstico del sector agropecuario y pesquero del Estado de Sonora (pp. 17-96). México: CIAD, SAGARHPA Y SAGARPA.

Samuelson, P. (1937). A note on measurement of utility. The Review of Economics Studies, 4(2), 155-161. doi: https://doi.org/10.2307/2967612

Servicio de Información Agroalimentaria y Pesquera (2018). Boletín de leche, enero-marzo. Recuperado de: http://infosiap.siap.gob.mx/opt/boletlech/ Bolet\%C3\%ADn\%2ode\%2oLeche\%20enero-marzo\%202018.pdf.

Strotz, R. (1956). Myopia and inconsistency in dynamic utility maximization. The Review of Economic Studies, 23(3), 165-180. doi: https:// doi.org/10.2307/2295722

Tanaka, T., Camerer, C. y Nguyen, Q. (2010). Risk and time preference: Linking experimental and household survey data from Vietnam. American Economic Review, 10o(1), 557-571. doi: https://oi. org/10.1257/aer.100.1.557

Thaler, R. (1981). Some empirical evidence on dynaic inconsistency. Economic Letters, 8, 201-207. doi: https://doi.org/10.1016/0165-1765(81)90067-7

Thaler, R. (1988). Anomalies: The winner's curse. Journal of Economic Perspectives 2(1), 191-202. doi: https://doi.org/10.1257/jep.2.1.191

Tversky, A. y Kahneman, D. (1974). Judgment under uncertainty: Heuristics and biases. Science, 185(4157), 1124-1131. doi: https://doi.org/10.1126/ science.185.4157.1124

Vanderveldt, A., Oliveira, L. y Green, L. (2016). Delay discounting: Pigeon, rat, human - does it matter? Journal of Experimental Psychology: Animal Learning and Cognition, 42(2), 141-162. doi: https://doi.org/10.1037/xanooooo97 